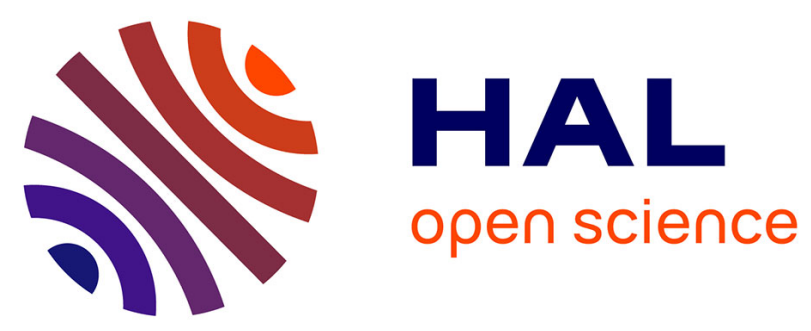

\title{
Urban-X: A Self-organizing Cognitive Wireless Mesh Network for Dense City Environments
}

\author{
Luciano Bononi, Wooseong Kim, Andreas J. Kassler, Marco Di Felice, Mario
}

Gerla

\section{To cite this version:}

Luciano Bononi, Wooseong Kim, Andreas J. Kassler, Marco Di Felice, Mario Gerla. Urban-X: A Self-organizing Cognitive Wireless Mesh Network for Dense City Environments. 9th Wired/Wireless Internet Communications (WWIC), Jun 2011, Vilanova i la Geltrú, Spain. pp.398-409, 10.1007/9783-642-21560-5_33. hal-01583638

\section{HAL Id: hal-01583638 \\ https://hal.inria.fr/hal-01583638}

Submitted on 7 Sep 2017

HAL is a multi-disciplinary open access archive for the deposit and dissemination of scientific research documents, whether they are published or not. The documents may come from teaching and research institutions in France or abroad, or from public or private research centers.
L'archive ouverte pluridisciplinaire HAL, est destinée au dépôt et à la diffusion de documents scientifiques de niveau recherche, publiés ou non, émanant des établissements d'enseignement et de recherche français ou étrangers, des laboratoires publics ou privés. 


\title{
Urban-X: A Self-Organizing Cognitive Wireless Mesh Network for Dense City Environments
}

\author{
Wooseong Kim ${ }^{1}$, Andreas J. Kassler ${ }^{2}$, Marco Di Felice ${ }^{3}$, Mario Gerla ${ }^{1}$, and \\ Luciano Bononi ${ }^{3}$ \\ 1 Department of Computer Science, University of California, Los Angeles, USA \\ 2 Department of Computer Science, Karlstad University, Karlstad, Sweden \\ 3 Department of Computer Science, University of Bologna, Bologna, Italy
}

\begin{abstract}
The practical deployment of Wireless Mesh Networks (WMNs) using unlicensed ISM band within dense urban scenarios is difficult due to the increasing number of wireless devices operating in those licensed exempt frequencies. For this reason, current research on WMN is directed towards novel and more flexible network paradigms which would allow the WMN to dynamically adapt to the environmental interference conditions. Here, we propose Urban-X, which is a novel cross-layer architecture for self-organizing WMNs over urban scenarios. Urban-X combines elements from classical Multi-Radio Multi-Channel (MC-MR) technology with novel Dynamic Spectrum Access (DSA) mechanisms. The self-organizing behavior is achieved through a novel distributed channel assignment scheme, an adaptive multi-path routing scheme and a flexible layer 2.5 channel and path scheduler algorithm. Based on the current interference on each channel, Urban-X performs channel allocation among the nodes of the WMN, updates the available paths towards the gateways and distributes the internal traffic among the paths/channels in order to maximize the network throughput while minimizing interference to the external networks. Simulation results demonstrate the effectiveness of our cross-layer approach in terms of increased throughput compared to traditional routing schemes for WMNs, and its adaptiveness to the variation in channel conditions and external user traffic.
\end{abstract}

Key words: Wireless Mesh Networks, Multi-Radio, Multi-Channel, CrossLayer Design, Performance Evaluation.

\section{Introduction}

Wireless Mesh Networks (WMNs) have emerged as a key technology for next generation wireless networks, mainly due to the possibility to provide broadband Internet access over urban scenarios at higher performance and lower cost than other wireless access technologies, e.g the 3G Mobile Internet [1]. Nowadays, several deployments of WMNs demonstrate the possibility to build multi-hop wireless networks which can cover highly dense urban areas (e.g Vienna, Frankfurt, etc) and serve thousands of users (e.g. the Chaska project [4]). However, the performance of WMNs is still not comparable with the wired Internet, due 
to interference problems in highly dense urban environments.

Multi-Radio Multi-Channel (MC-MR) technology constitutes a well-investigated approach to alleviate the interference problem in WMNs, by multiplying the spectrum resources available to each mesh Access Point (AP) [3, 15]. However, most of the previous works address the problem of self-interference (i.e. interference between the nodes of the WMN) by assigning adjacent APs to orthogonal channels. We highlight here that the interference problem becomes much more serious when the WMN nodes must share the spectrum with external devices, such as residential Wi-Fi networks, which belong to different organizations [11]. In this paper, we call such devices Primary Nodes (PNs) to distinguish from the nodes of the WMN. In such a case, Channel Assignment (CA) schemes should consider the dynamic changes of the PN interference. Moreover, a more flexible network design is required to dynamically reconfigure the CA and traffic forwarding process based on the actual environmental conditions [19].

In this paper, we combine elements from classical MC-MR technology with Dynamic Spectrum Access (DSA) [2] mechanisms to deploy self-organizing WMNs in high dense city environments. To this aim, we propose a novel cross-layer architecture, called Urban-X, which allows a WMN to co-exist with other PNs, and to dynamically adapt its configuration in order to $(i)$ maximize its performance while (ii) minimizing the impact to the PNs. In Urban-X, self-organization is achieved through the mutual integration of four main components:

- Spectrum Sensing: In contrast to classical cognitive radio networks [2], Urban$\mathrm{X}$ is deployed in ISM band. Thus, a WMN node does not need to vacate its channel even if PN traffic is detected. However, we use sensing techniques to implement novel collaborative load estimation mechanisms, through which the WMN nodes can estimate the PN workload on each channel.

- Channel Assignment: While existing CA algorithms mainly consider intramesh interference [12], Urban-X CA (UCA) uses input from the collaborative spectrum sensing to balance the impact of interference caused by PNs with intra- and inter-flow interference to achieve robustness and high performance.

- Multipath Routing: Urban-X leverages the availability of multiple paths towards the gateways by maintaining a forwarding mesh centered around the best metric path. The routing protocol extends AODV by maintaining a set of candidate forwarders at each node for each source/destination pair. In contrast to previous works [6][9], our approach can dynamically change individual path segments on a per-packet basis to effectively cope with interference caused by variation in PN traffic and intra-mesh congestion.

- Forwarding and Scheduling: Urban-X develops novel packet scheduling algorithms for selecting a route and a channel in the multipath WMN. Although a practical approach based on back-pressure scheduling [5][10][18] has been developed in a single radio WMN [14], Urban-X is the first work that extends it to MC-MR WMNs by also taking into account the channel switching cost.

Using a detailed evaluation of Urban-X in the ns-2 simulator, which has been extended in [7] to model MC-MR operation and PN traffic, we demonstrate that Urban-X is able to dynamically adapt the WMN configuration to the variation of 
channel conditions and external user traffics, thus achieving higher performance than classical CA and single-path routing schemes for MR-MC WMNs.

The paper is organized as follows. In Section II, we review existing CA and routing schemes for MR-MC WMNs. Section III describes our Urban-X architecture, along with our channel assignment, routing and scheduling algorithms. Evaluation results are shown in Section IV. The paper concludes in Section V.

\section{Related Works}

Most of the current research on WMNs focuses on techniques to enhance the network capacity through channel diversity in the $2.4-5 \mathrm{GHz}$ bands and multi-radio technology [12][17]. Generally speaking, Channel Assignment (CA) for multiple radio interfaces on a conflict graph is a NP-hard problem, and several CA schemes have been proposed in the literature, usually based on static, semidynamic or hybrid approaches. In static CA approaches, the binding between channel and radio interface is decided during the network setup [10]. In semidynamic CA approaches, the nodes change a static assignment over time (e.g. on a minute or hour timescale) to cope with changes in environment [3][15]. However, both the static and the semi-dynamic approaches are not feasible for urban environments where traffic demands and interference conditions can change rapidly like in Urban-X. To avoid the ripple effect of dynamic and semi-dynamic channel assignment, hybrid solutions have been proposed (e.g. NetX [12]). Here, each node is equipped with a receiving interface using a fixed channel and a switchable interface which dynamically change channel to send data to the neighbours' fixed interfaces. However, the CA scheme in [12] does not address asymmetric traffic and interference caused by PNs.

At network layer, many interference-aware routing metrics have been proposed for WMNs, e.g. the expected transmission count (ETX) [6], the expected transmission time (ETT) $[8]$ and the weighted cumulative expected transmission time (WCETT) [9], among others. The main drawback of these metrics is that a given minimum metric path might become suboptimal over time due to varying traffic and link quality conditions. In addition, it is hard to quantify the impact of external PN traffic using such metrics. In this paper, we develop routing and channel switching scheduling schemes which use local information to approach the performance of the centralized schemes as an upper bound. The scheduling scheme is based on a back-pressure algorithm with differential backlogs [18] of the multiple paths, which solves a network utility maximization problem [5][10]. For multi-channel, multi-radio (MC-MR) WMNs, the authors of [13][16] proposed heuristic algorithms of maximal weight scheduling in a distributed manner but requiring heavy signaling traffic for its implementation. Horizon [14] proposed a simplified system model which allows to estimate the path quality for backpressure scheduling with less probing overhead. However, it works for single radio WMNs and considers only congestion delay for the path cost. Here, we extend the framework in [14] for MC-MR WMNs, taking also into account the impact of channel switching overhead in the scheduling process. 


\section{Urban-X architecture}

\subsection{System Model}

The Urban-X architecture is shown in Figure 1(a). Urban-X consists of Mesh Clients (MCs) and Cognitive Mesh Nodes (CMNs) that coexist with PNs. The PNs can be residential 802.11 Access Points (APs) as well as Bluetooth or Zigbee devices. In dense urban areas, experimental results show that these devices already occupied most of the spectrum in ISM bands [11]. The CMNs are wireless routers equipped with three WiFi radio interfaces (i.e. R1, R2 and R3), through which they form a forwarding backbone as in Figure 1(a). R1 and R2 are used to receive or transmit packets simultaneously on different ISM channels, while $\mathrm{R} 3$ is used for exchanging control messages. The R1 is tuned to a semi-dynamic channel that changes according to PN activity and mesh network traffic. The CA strategy for R1 is described in Section 3.3. The R2 is dynamically switching among the channels of the neighbor CMNs with a predefined switching interval (e.g. $40 \mathrm{msec}$ ). In current platforms, switching delay is the overhead to switch channels on a per-packet basis [12], and it is approximately $1 \mathrm{msec}$. Compared to existing MC-MR solutions [3,15], the hybrid multi-radio approach of Urban-X provides two main benefits: (i) it achieves high throughput using a CA strategy which takes into account the impact of both internal and external interference (Section 3.3), and (ii) it preserves the network connectivity through the switchable transmitting interface. In addition, we use a designated interface for control (R3), which is fixed to a common control channel (CCC) to disseminate routing information and updates of the CA protocol. Such a control interface introduces an additional cost which can be justified by the overhead reduction for broadcast messages, which otherwise would require to send multiple copies of the same packets on all the available channels, as in [12]. The channel selection for the CCC is out of the scope of this work. An example of operations of the Urban-X CA scheme in a three-nodes chain topology is shown in Figure 1(b).

\subsection{Spectrum Sensing and Primary Traffic Load Estimation}

An important aspect of Urban-X is the effective avoidance of external interference due to PN traffic. This is achieved by the interference aware CA scheme described in Section 3.3, which requires information on external interference. Therefore, the CMNs sense the spectrum periodically and estimate the channel workloads of PN traffics $(\omega)$, defined as the ratio of time the channel is used by an active PN. In Urban-X, each CMN performs spectrum sensing through an energy detector scheme, which detects a signal during a single symbol duration (e.g. $4 \mu$ sec in case of 802.11a). The reliability of the estimated results depends on the sensing period and on the sampling rate. As the sensing period is longer and the sampling rate is high, the result is more reliable. According to our simulation study, more than $300 \mathrm{msec}$ for the sensing period is required to get an approximated workload value with $10 \%$ error. In addition, estimating the busy or 


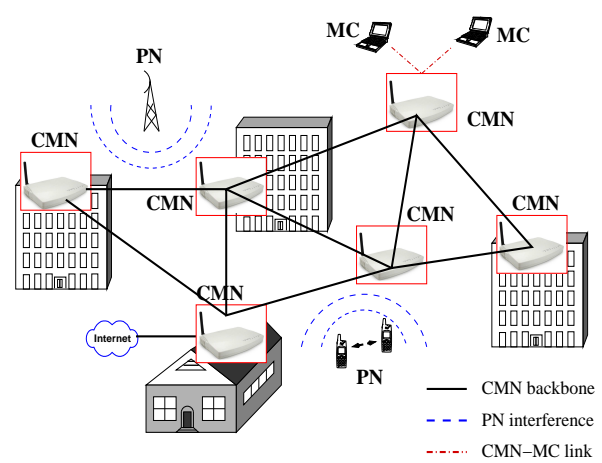

(a)

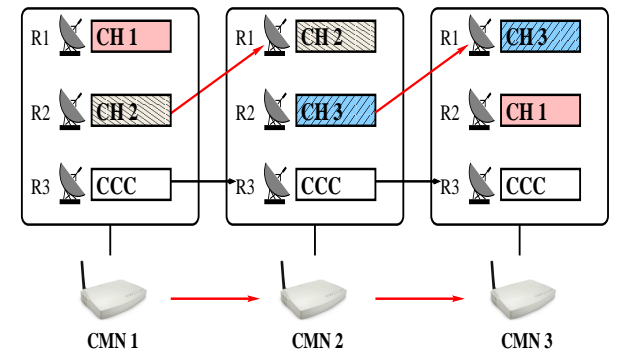

(b)

Fig. 1. The Urban-X architecture is shown in Figure 1(a). An example of operations of the Urban-X CA scheme is shown in Figure 1(b).

idle durations needs a sampling rate that at least is higher than the Nyquist sampling rate (e.g. 0.3, $0.5 \mathrm{msec}$ ). Thus, each CMN calculates the channel workload as: $\omega=\frac{T_{\text {busy }}}{T_{\text {busy }}+T_{\text {idle }}}$ where $T_{\text {busy }}\left(T_{\text {idle }}\right)$ represents the average time the channel is detected busy (idle) during the sensing period. The estimation of $\omega$ poses at least three important issues to be solved for its practical implementation. First, a CMN is not allowed to send packets during the second period because it cannot distinguish between PN and CMN traffics. Second, a long sensing period for each channel (e.g. 50, 100, $200 \mathrm{msec}$ ) would significantly reduce the network performance. Third, with increasing number of available channels, a significant time would be required for each CMN to estimate the PN traffics on all the channels. To solve the first issue, we assume that sensing is synchronously performed by the CMNs, leveraging the CCC for synchronization issues [2]. To reduce the impact of sensing interval on network performance, we use collaborative sensing techniques, i.e. each CMNs exchange sensing information with neighbors to enhance the measurement performance. Since channel status is asymmetric in receive and transmit nodes, measuring $\mathrm{PN}$ workload should be executed on both the fixed channel used by $\mathrm{R} 1$ and on the transmitting channel of $\mathrm{R} 2$. The details of our collaborative sensing scheme can be found in [20].

\subsection{Interference Aware Channel Assignment}

In Urban-X, the CA scheme defines the channel to be used on the fixed radio $\mathrm{R} 1$. In our CA scheme, we take into account the impact of external interference (caused by PNs) and internal interference (caused by other CMNs). For this purpose, each CMN chooses the channel which has the least impact by PN traffics while at the same time minimizing internal interference caused by CMN traffics along the path to the receiver node (intra- and inter flow interference). Algorithm 1 shows the operations of the Urban-X CA scheme (UCA). 


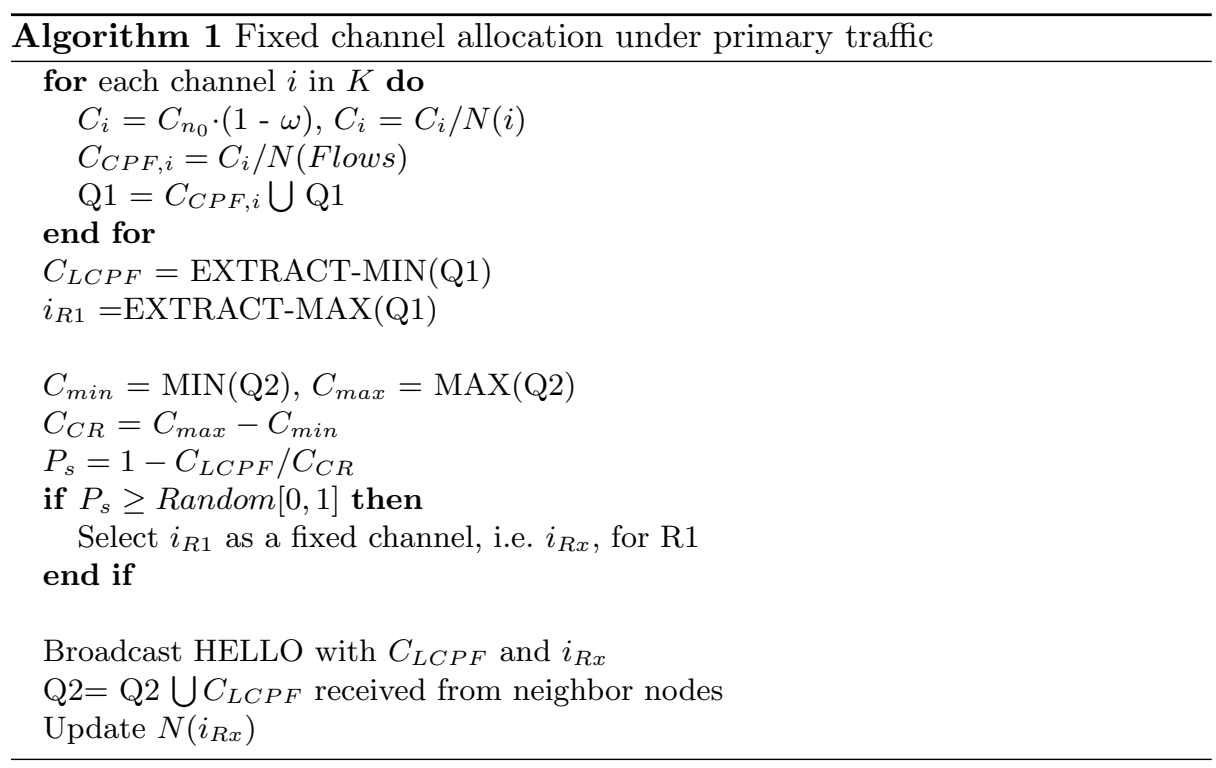

From the results of channel workload measurement $\omega$ in the previous section, we can approximately derive an expected capacity of channel $i$ as: $C_{i}=C_{n_{0}}$. $(1$ $\omega)$ where $C_{n_{0}}$ denotes the non-interfered channel capacity as given by Shannon under Additive White Gaussian Noise (AWGN). For each channel $i$, the CMNs estimate the available Capacity per Flow, i.e. $C_{C P F, i}$, by dividing $C_{i}$ first for the the number of nodes selecting channel $i$ within two hop neighborhoods, i.e. $N(i)$, and then for the number of active flows i.e. $N($ Flows). Then, the maximum capacity channel $i_{R 1}$ is chosen for the R1 interface with a probability $P_{s}$. As shown in Algorithm 1, the value of $P_{s}$ is a function of the Least Capacity per Flow, i.e. $C_{L C P F}$, of the neighbor nodes, so that a node serving many flows compared to its link capacity or suffering heavy external interference from PNs gets prioritized in the CA. Then, each CMN periodically broadcasts an HELLO messages including the $C_{L C P F}$ value and the selected channel for R1 (i.e. $i_{R x}$ ) on the R3 interface. When receiving an HELLO message from other nodes, each CMN stores the received information in a neighborhood information table.

\subsection{Multipath based Forwarding Mesh Structure}

Multipath routing protocols enable CMNs to discover multiple paths towards the gateway and to use alternate paths when the current one is no longer available or congested due to PNs or CMNs traffic. In addition, load-balancing schemes allow to distribute the traffic among the available paths, considering the performance of each path. Multipath routing is therefore an efficient method to cope with varying PN traffic dynamics. However, the existing routing metrics for WMN, e.g. ETX [6], ETT [8] or WCETT [9] are not appropriate for Urban-X since an established path could rapidly change its quality due to the dynamic 
PN activities. For this reason, Urban- $X$ creates an adaptive forwarding mesh structure in which a forwarding node maintains multiple next hop candidates for a given destination. Packets are distributed over the next-hop candidates based on the individual path and link conditions, through the scheduler scheme described in Section 3.5. Thus, a packet will traverse just one path that however may change dynamically at every hop. A detailed procedure of our multipath routing protocol is illustrated in Figure 2(a). We have extended the popular AODV routing scheme for multipath operations. In our scheme, a source node broadcasts a route request (RREQ) message through the CCC on the R3 interface. The intermediate nodes rebroadcast the RREQ messages received from different upstream nodes. Unlike AODV, an intermediate node can forward duplicate RREQ messages if they contain a lower metric value than the one stored in the routing table. We will discuss the routing metric (Cumulative ETT) in the next section separately. After receiving multiple RREQ messages, a destination node replies with multiple route reply (RREP) messages by unicasting. In order to limit the forwarding mesh structure, the forwarding mesh is constructed of individual paths that are not longer than a certain hop count. The destination node calculates the Threshold hop count, ThresholdHC $=\alpha \cdot$ hop $_{\min }$ for the RREP message in which $\alpha \geq 1$ and hop min is the hop count of the shortest path that has the lowest metric among the multiple RREQ messages. Intermediate nodes become forwarders of a given flow only when the hop count from them to the source is less than the ThresholdHC value. Otherwise they discard the RREP message and remove the routing entry of the flow. While a broader mesh structure (by e.g. setting $\alpha=2$ ) enhances spatial diversity, it also leads to longer paths and inter-flow interference. Figure 2(a) depicts an example with $\alpha=1$. The details of the Urban-X routing scheme can be found in [19].

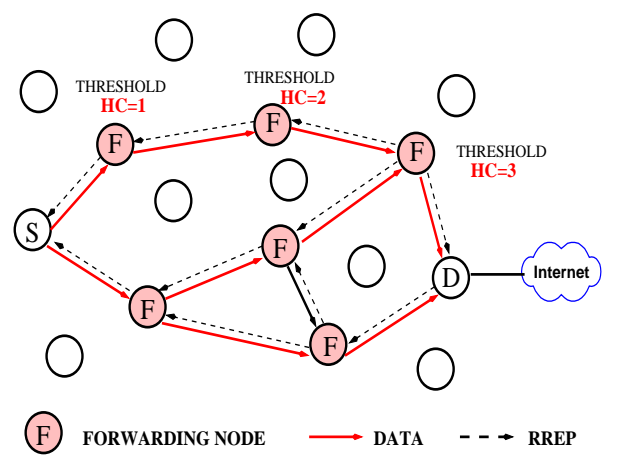

(a)

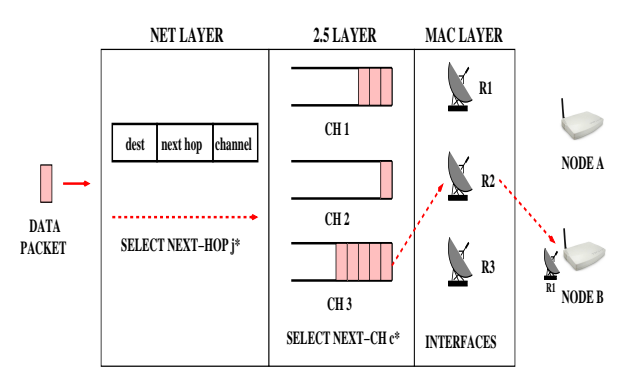

(b)

Fig. 2. The Urban-X forwarding mesh is shown in Figure 2(a). The packet and channel scheduler operations are shown in Figure 2(b). 


\subsection{Routing and Channel Switching Scheduling}

Since in Urban- $X$ each CMN can choose among multiple next hop candidates, we have developed a routing scheduler, which decides the next hop candidate for every packet to be transmitted. To this aim, we have proposed a new layer 2.5 which maintains channel queues that hold packets to be sent on the corresponding channels of the switchable interface R2. Moreover, we have developed a channel scheduler at the layer 2.5 that decides the channel of the interface R2 for the next switching interval. Figure 2(b) shows the operations of the packet and channel scheduler. The channel scheduler is based on the Horizon framework [14], which defines a simplified model for back-pressure scheduling in a single channel WMN. In Urban-X, we have extended the model in a MR-MC environment also considering the channel switching cost. We have formulated the channel scheduling problem as an optimization problem where each CMN must determine the best channel which maximizes the utility function, i.e. throughput. Due to space shortage, we omit the details of the optimization framework, which can be found in [19]. Instead, we report here only our heuristic solutions to the scheduling problem. As shown by Figure 2(b), our scheduling algorithm selects the next hop $j^{*}$ which is the minimum cost path in the routing layer and the channel $c^{*}$ which has the largest number of flow packets, $P_{c}^{f}$ to maximize the packet transmission in layer 2.5:

$$
j^{*}=\arg \min _{j \in G} C_{\text {rate }, j}^{f}, \quad c^{*}=\arg \max _{c \in K} \frac{P_{c}^{f}}{R}
$$

The path and channel costs for the scheduling algorithms are derived in Eq.(2) and (3).

$$
\begin{gathered}
C_{c h, i}^{f}=\max \frac{P_{i}^{f}}{z_{c}}+C_{\text {rate }, j}^{f} \\
C_{\text {rate }, j}^{f}=\max _{c \in K} \frac{\operatorname{Exp}\left(P_{c}^{f}\right)}{R}+C_{c h, j}^{f}
\end{gathered}
$$

where the path cost, $C_{\text {rate }, j}^{f}$ refers to the packet transmission delay of a flow via a node $j$ to a destination. In addition to the path cost, channel cost, $C_{c h, i}^{f}$ at node $i$ denotes the additive delay from channel switching under the virtual link rate in Eq.(2). The total path cost, $C_{p}^{f}=C_{c h, s}^{f}$ from a source node is utilized for the routing metric $\left(C E T T_{p(s, d)}=C_{p}^{f}\right)$ in the multipath routing protocol as well. The virtual link rate can be derived as $R \cdot T_{\text {slot }, c} / T_{\text {super }}$ in which $T_{\text {slot }, c}$ is the number of slots for the corresponding channel $c$ in a single super frame $T_{\text {super }}$ that has at least one time slot for every active channel to prevent starvation. Moreover, virtual data rate includes the additional delay $D_{s w}$ for channel switching when the currently transmitting channel is not $c$. As shown in Eq.(3), the path cost considers the actual data rate $R$ and the expected number of queued packets, $\operatorname{Exp}\left(P_{c}^{f}\right)=P_{c}^{f} \cdot\left(1-P E R_{i, j}\right)$ for depressing queueing over the link to node $j$ on channel $i$, which has an estimated Packet Error Rate $P E R_{i, j}$. Assuming a steady state configuration, our heuristic can be proved to maximize the network utility 
by showing that it satisfies the optimality conditions defined in [14]. Details of the proof and of the scheduler implementation can be found in [19].

\section{Performance Evaluation}

We have implemented Urban-X in ns2 with extensions from [7] to model the PN activity and MC-MR operations. Each CMN is equipped with three radio interfaces using $2 \mathrm{Mbps}$ data rate without rate adaptation. Each radio interface can use in total 11 orthogonal channels, but channel 1 is designated for the implementation of the CCC. We plan to extend the analysis to cope with adjacent channel interference (ACI) as future work. The sensing period for the workload measurement is $70 \mathrm{msec}$ per second. We report here the results for a random topology scenario, composed by $50 \mathrm{CMNs}$ randomly placed inside an area of $1000 \mathrm{~m}$ by $1000 \mathrm{~m}$. Three CBR flows transmit data with varying data rates from 200 to $1000 \mathrm{Kbps}$ between random source-destination pairs. We varied the number of active PNs in the scenario, and the workload $\theta$ produced by each PN. The PN traffic is modeled through a classic birth-death Markovian model [2] with parameters $\lambda$ and $\mu$. Based on such model, the Cumulative Distribution Functions (CDFs) for the expected idle duration, i.e. $T_{\text {idle }}$, and busy duration, i.e. $T_{\text {busy }}$, can be derived as:

$$
P\left(T_{\text {idle }}<t\right)=1-e^{-\lambda t}, \quad P\left(T_{\text {busy }}<t\right)=1-e^{-\mu t}
$$

In the following, we propose a modular evaluation of Urban-X. In Section 4.1, we analyze the performance of our CA scheme under different PN workloads and CMNs traffic [19]. In Section 4.2, we investigate the benefit provided by the multi-path forwarding mesh over a single-path approach. We compute the network throughput and the end-to-end delay as performance metrics [20].

\subsection{Channel Assignment Analysis}

In Figures 3(a), 3(b) and 3(c) we compare the performance of the Urban-X CA scheme (UCA) described in Section 3.3 against the performance of the Distributed Channel Assignment (DCA) scheme described in [12]. In DCA, the number of neighbor nodes on a given channel is balanced within the two hop neighborhood. However, DCA does not consider the impact of external PN traffic on the channel selection process. Figure 3(a) shows the aggregated network throughput as a function of the load produced by each flow (on the $x$-axis). We consider a scenario with 20 active PNs distributed on 10 channels, and two configurations of PN workload (20\% and 60\%). As shown in Figure 3(a), the throughput of UCA and DCA is close under low traffic load and PN workload, while UCA can achieve higher performance when the PN interference increases. Under high system load (i.e. $1000 \mathrm{~Kb} / \mathrm{s}$ ), UCA provides a performance improvement of $20 \%$ for $\theta=20 \%$, and of more than $60 \%$ for $\theta=60 \%$, thus confirming the importance of PN detection. Figure 3(b) shows the aggregated network throughput when we vary the system load (on the $x$-axis) and the number of channels 


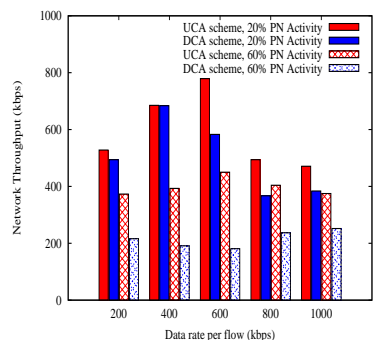

(a)

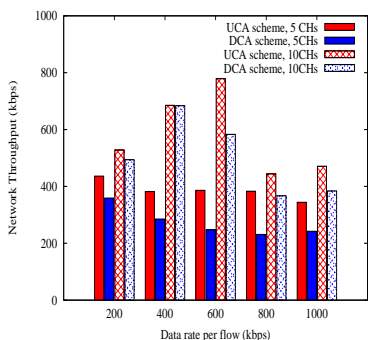

(b)

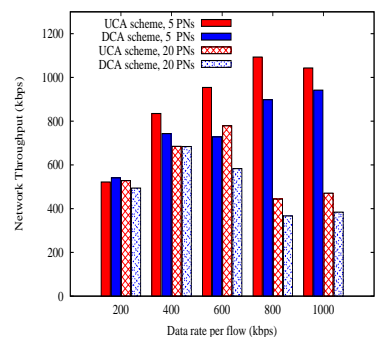

(c)

Fig. 3. The UCA and DCA throughputs as a function of the system load produced by the CMNs are shown in Figures 3(a), 3(b) and 3(c).

(i.e. 5 or 10). We consider a scenario with 20 active PNs with $20 \%$ workload. We observe that the number of occupied channels due to PN activity is more critical to network performance than workload of a given PN population since throughput difference between workloads in Figure 3(a) is smaller compared to Figure 3(b). Figure 3(c) confirms that UCA is able to achieve higher throughput than DCA also when we vary the number of active PNs in the scenario.

\subsection{Routing Analysis}

In order to evaluate the ability of our architecture to cope with dynamic variation of the PN traffic, we have considered two different configurations of Urban-X:

- Urban-X with single-path routing (UCA-SP): we consider the basic Urban-X architecture with the CA scheme described in Section 3.3, but with the multipath (Section 3.4) and channel scheduling (Section 3.5) modules disabled. A single path is used between the source and destination nodes.

- Urban-X with multi-path routing (UCA-MP): we consider the full Urban-X architecture, with the multipath and channel scheduling modules enabled. Multiple paths are used between the source and destination nodes.

The main goal of the simulation analysis is to show that UCA-SP cannot maximize network throughput because of fluctuations in PN traffics, while UCAMP can increase robustness and thus achieve higher performance through the adaptive path and channel scheduler over the multipath mesh. Figure 4(a) shows the aggregated network throughput as a function of the load produced by each flow (on the $x$-axis), for two configurations of the PN workload (20\% and $60 \%$ ), produced by $5 \mathrm{PNs}$. Figure 4(b) shows the same analysis when we vary the system load and the number of active PNs ( 5 and 20), with a PN workload of $20 \%$. UCA-MP outperforms UCA-SP in almost every scenario under varying number of PNs and workloads as shown in Figure 4(a) and Figure 4(b) except for the configuration with $200 \mathrm{Kbps}$ data rate where the advantages of the multi-path are penalized by the higher overhead and the additional self interference effect. 


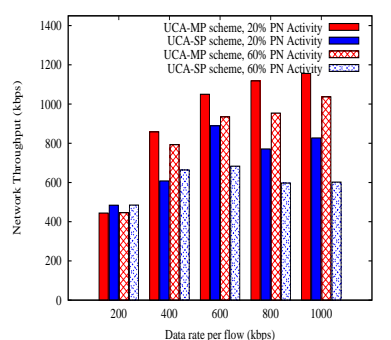

(a)

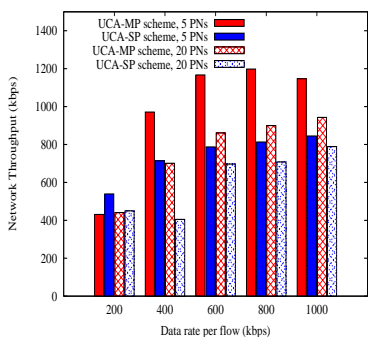

(b)

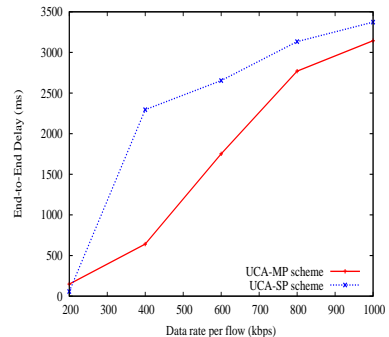

(c)

Fig. 4. The UCA-SP and UCA-MP throughputs as a function of the system load produced by the CMNs are shown in Figures 4(a), 4(b) and 4(c).

Interestingly, the aggregated throughput of UCA-MP increases with increasing data rate, which indicates that UCA-MP can effectively reduce the inter-flow interference. Figure 4(c) shows the end-to-end delay as a function of the systemload (on the $x$-axis) for 5 PNs , PN workload of 20\%. and 10 channels. For low data rate, switching delay becomes more critical since network congestion is not severe and the number of PNs are scarce in the network. As a result, UCA-MP shows longer delay at $200 \mathrm{Kbps}$ compared to UCA-SP. However, as the number of PNs and data rate of flows increases, UCA-MP considerably reduces the endto-end delay compared to UCA-SP.

\section{Conclusions}

In this paper, we have presented Urban-X, a novel cross-layer architecture which combines elements from classical MC-MR technology with Dynamic Spectrum Access principles to deploy self-organizing WMNs over urban environments. To this purpose, we have proposed a novel CA scheme which takes into account the impact of external interference on each channel and we have integrated it with a multipath routing scheme and a back-pressure channel/packet scheduler, through which we are able to dynamically distribute the traffic inside the WMN based on actual channel conditions. The simulation results reveal that Urban$\mathrm{X}$ can increase robustness and performance significantly under a wide range of scenarios. Future works include: the implementation of the UCA scheme on a testbed and the analysis of TCP performance over Urban-X.

\section{Acknowledgments}

This research is supported by grant YR2009-7003 from Stiftelsen för internationalisering av högre utbildning och forskning (STINT). 


\section{References}

1. I. F. Akyildiz, X. Wang, and W. Wang. "Wireless Mesh Networks: A Survey,", Computer Networks Journal, 47(4), pp. 445-487, 2005.

2. I. F. Akyildiz, W. Y. Lee, M. C. Vuran, and S. Mohanty. "NeXt Generation/Dynamic Spectrum Access/Cognitive Radio Wireless Networks: A Survey, “ Computer Networks Journal, 50(1), pp. 2127- 2159, 2006.

3. M. Alicherry, R. Bhatia, and L. E. Li, "Joint channel assignment and routing for throughput. optimization in multi-radio wireless mesh networks," in Proc. of ACM MOBICOM, 2005.

4. Chaska wireless solutions. http://www.chaska.net/.

5. M. Chen, S. Low, M. Chiang, and J. Doyle, "Cross-layer congestion control, routing and scheduling design in ad hoc wireless networks," in Proc. of IEEE INFOCOM, 2006.

6. D. Couto, D.Aguayo, J.Bicket, and R.Morris, "A high-throughput path metric for multi-hop wireless routing," in Proc. of ACM MOBICOM, 2003.

7. M. Di Felice, K. R. Chowdhury and L. Bononi, "Modeling and performance evaluation of transmission control protocol over cognitive radio ad hoc networks," in Proc. of ACM MSWIM, 2009

8. R. Draves, J.Padhye, and B.Zill, "Comparison of routing metrics for static multihop wireless network," in Proc. of ACM SIGCOMM, 2004.

9. R. Draves, J.Padhye, and B.Zill, "Routing in multi-radio, multi-hop wireless mesh networks," in Proc. of ACM MOBICOM, 2004.

10. L. Georgiadis, M. Neely, and L. Tissiulas, "Resource allocation and cross-layer control in wireless networks," Foundation and Trends in Networking, 1(1), pp. 1-144, 2006.

11. D. Gokhale, S. Sen, K. Chebrolu, and B. Raman, "On the feasibility of the link abstraction in (rural) mesh networks," in Proc. of IEEE INFOCOM, 2008.

12. P. Kyasanur and N. Vaidya, "Routing and link-layer protocols for multi-channel multi-interface ad hoc wireless networks," ACM MC2R, 10(1), pp. 31-43, 2006.

13. S. Merlin, N. Vaidya, and M. Zorzi, "Resource allocation in multi-radio multichannel multi-hop wireless networks," in Proc. of IEEE INFOCOM, 2008.

14. B. Radunovic, C. Gkantsidis, D. Gunawardena, and P. Key, "Horizon: Balancing tcp over multiple paths in wireless mesh network," in Proc. of ACM MOBICOM, 2008.

15. A. Raniwala and T. Chieueh, "Architecture and algorithms for an ieee 802.11-based multi-channel wireless mesh network," in Proc. of IEEE INFOCOM, 2005.

16. S. Rasool and X. Lin, "A distributed join channel assignment scheduling and routing algorithm for multi-channel ad hoc wireless networks," in Proc. of IEEE INFOCOM, 2007

17. J. So and N. Vaidya, "Multi-channel mac for ad hoc networks: handling multichannel hidden terminals using a single transceiver," in Proc. of $A C M M O B I H O C$, 2004 .

18. L. Tassiulas and A. Ephremides, "Stability properties of constrained queueing systems and scheduling policies for maximum throughput in multihop radio networks," IEEE Transaction on Automatic Control, 37(12), 1992.

19. W. Kim, A. J. Kassler, M. Di Felice and M. Gerla, "Cognitive multi-radio mesh networks on ISM bands: a cross-layer architecture," in Proc. of IEEE IPCCC, 2010.

20. W. Kim, A. J. Kassler, M. Di Felice and M. Gerla, "UrbanX: towards distributed channel assignment in cognitive multi-radio mesh networks," in Proc. of IFIP Wireless Days, 2010. 